\title{
Primary tumor size thresholds in stage IA testicular seminoma: Implications for adjuvant therapy after orchiectomy and survival
}

\author{
Mounsif Azizi, MDa ${ }^{a}$, Charles C. Peyton, MDa ${ }^{a}$, David C. Boulware, MS $^{b}$, Scott M. Gilbert, MD, \\ MS $^{a, c}$, Wade J. Sexton, MD ${ }^{a,{ }^{*}}$ \\ aDepartment of Genitourinary Oncology, H. Lee Moffitt Cancer Center and Research Institute, \\ Tampa, FL \\ bepartment of Biostatistics, H. Lee Moffitt Cancer Center and Research Institute, Tampa, FL \\ 'Department of Health Outcomes and Behavior, H. Lee Moffitt Cancer Center and Research \\ Institute, Tampa, FL
}

\section{Abstract}

Objectives: Primary tumor size (PTS) is the main prognostic factor for relapse in clinical stage (CS) IA testicular seminoma (T1NOMOS0) and the 8th edition of the Tumor-Node-Metastasis staging system now subcategorizes pT1 tumors into pT1a and pT1b based on PTS ( $<3 \mathrm{~cm}$ and $\geq 3$ $\mathrm{cm}$, respectively). We attempted to assess PTS as a prognosticator for overall survival (OS) in CS IA seminoma and to evaluate the comparative effectiveness of active surveillance (AS) versus adjuvant therapy (AT) in patients with large primary tumors (LPT).

Methods and materials: In the National Cancer Database (2004-2014), 2455 (47.7\%) and 2685 (52.3\%) patients with CS IA seminoma were treated with AS and AT, respectively. AT was defined as the receipt of chemotherapy or radiation within 3 months after orchiectomy. A cut-point analysis was performed to determine the optimal PTS threshold predicting OS at 5 years after orchiectomy. Inverse-probability of treatment weighting (IPTW)-adjusted Kaplan-Meier curves and Cox regression analyses were used to compare OS of patients with LPT (using the optimal PTS cut-point) treated with AS versus AT.

Results: In adjusted analysis, pathologic T-stage (pT1a vs. pT1b) did not predict OS and no OS benefit was noted in pT1b patients treated with AT. The optimal PTS cut-point was $4.5 \mathrm{~cm}$. In multivariable analysis, patients with LPT ( $₫ 4.5 \mathrm{~cm}$ ) had an increased risk of overall mortality (HR $=1.87, P=0.003)$. Kaplan-Meier curves revealed that OS was superior in patients with LPT treated with AT (IPTW-adjusted log-rank $P=0.029$ ). In IPTW-adjusted Cox regression analysis, AT was associated with an OS benefit in patients with LPT (HR $=0.59,95 \%$ CI: $0.39-0.91, P=$ $0.017)$.

\footnotetext{
*Corresponding author. Tel.: +1-813-745-3973; fax: +1-813-745-8494. Wade.Sexton@moffitt.org (W.J. Sexton). Conflict of interest None.

Supplementary materials

Supplementary material associated with this article can be found in the online version at https://doi.org/10.1016/j.urolonc.2019.09.022.
} 
Conclusions: In this National Cancer Database analysis, PTS was a predictor of OS in CS IA seminoma. An OS benefit was noted for individuals with LPT (defined as PTS $\geq 4.5 \mathrm{~cm}$ ) managed with AT. These findings may warrant refinement of Tumor-Node-Metastasis staging system.

\section{Keywords}

Testicular neoplasms; Seminoma; Prognostic factors; Observation; Adjuvant therapy

\section{Introduction}

Testicular cancer is an uncommon malignancy that accounts for less than $1 \%$ of cancers in men in the United States [1]. Yet, with 9,310 new cases estimated in 2018, it remains the most common solid tumor diagnosed among men aged 20 to 34 years. Survival outcomes are overall excellent in testicular seminoma, the most common type of testicular germ cell tumor [1,2]. In clinical stage (CS) I seminoma patients, 5-year disease-specific survival from centers of excellence reaches $99 \%$ while 5-year relapse rates range between 10-20\% after orchiectomy $[3,4]$.

Two prognostic factors are used to identify patients at greater risk of relapse and guide decision-making regarding adjuvant therapies in CS I seminoma: primary tumor size and rete testis invasion [5,6]. Patients with one or both of these risk features are at a higher risk of relapse compared to those without risk factors [4]. A primary tumor size $\geq 4 \mathrm{~cm}$ was first identified as a risk factor for relapse in a surveillance series reported in the early 1990s, and although widely studied, its predictive power remains unclear [4,7-16]. Other size thresholds including 3, 6 and 7-cm have also been examined [8,14,17-19]. The 8th edition of the American Joint Commission on Cancer (AJCC) Cancer Staging Manual now subcategorizes CS IA (pT1cN0M0S0) pure seminoma into pT1a and pT1b based on a $3-\mathrm{cm}$ primary tumor size cut-point [20].

Most CS IA seminoma patients with pT1a and pT1b primary tumors are cured with orchiectomy alone and will not relapse [12]. Due to the favorable prognosis of CS IA disease, management has shifted to a non-risk-adapted surveillance strategy [21]. Nonetheless, clinical management of seminoma patients with risk features such as large primary tumor size remains controversial [5]. The primary endpoint of the study was to assess primary tumor size as a prognosticator for overall survival (OS) using the National Cancer Data Base (NCDB). Secondary endpoints were to determine an optimal primary tumor size cut-point that predicts 5-year OS following radical orchiectomy and to evaluate the comparative effectiveness of active surveillance (AS) vs. adjuvant therapy (AT) for patients with large primary tumors.

\section{Material and methods}

\subsection{Data source and patient selection}

The NCDB is a hospital-based registry established by the Commission on Cancer (CoC), the American College of Surgeons and the American Cancer Society that captures approximately $70 \%$ of newly diagnosed malignancies in the United States each year at more 
than 1,500 participating CoC-accredited institutions [22]. Institutional review board approval was not required for the study in accordance with institutional regulation when dealing with deidentified collected data.

Data was extracted on 61,167 patients aged 18 years or older diagnosed with testicular cancer between 2004 and 2014. Using the 3rd edition of the International Classification of Diseases for Oncology (ICD-O) codes, we identified 33,818 patients with testicular seminoma (codes 9061-9063). Only individuals with AJCC CS IA (pT1N0M0S0) pure seminoma (code 9061$)$ were considered $(N=5,554)$. Patients with missing primary tumor size information were excluded $(N=209)$. A total of 5,345 individuals met the study criteria. Patients were grouped according to pathologic T stage (pT1a and pT1b) by using the $3 \mathrm{~cm}$ cut-point from the 8th edition of the AJCC Tumor-Node-Metastasis (TNM) staging system. pT1 patients with available treatment strategy data were further dichotomized into AS vs. AT groups (Fig. 1).

\subsection{Study measures, definitions, and outcomes}

The primary endpoint was OS from the date of initial testicular cancer diagnosis to the date of death or last follow-up. Treatment modalities were categorized into AS (observation) and AT (chemotherapy or radiation within 3 months after orchiectomy). The AT group is unlikely to include early salvage therapy cases since the treatment information provided in the NCDB is limited to the initial treatment course (before disease progression or recurrence).

Patient-level and socioeconomic variables included years of diagnosis, age at diagnosis, race, Charlson-Deyo score, insurance type, county type, income level, and education level. Facility covariates included distance from residence, location and type. Pathologic features included primary tumor size and surgical margins status.

\subsection{Statistical analyses}

The $t$ test and the $\chi^{2}$ test were used to compare continuous and categorical variables, respectively. Medians and interquartile ranges were reported for continuous variables. Categorical variables were reported as frequencies and proportions. Temporal treatment trends for CS IA seminoma were estimated with annual percent change (APC) in the receipt of AT vs. AS by using linear regression. Univariable and multivariable logistic regression analyses were used to identify independent predictors of AT receipt.

Unadjusted Kaplan-Meier curves and log-rank testing were used to compare OS in CS IA seminoma patients stratified by pathologic T stage (pT1a vs. pT1b) and to compare OS in pT1b patients stratified by treatment strategy (AS vs. AT). Univariable and multivariable Cox regression analyses were performed to determine independent predictors of OS (using backward elimination).

An optimal cut-point value for primary tumor size was then determined by methods previously described by Contal and O'Quigley [23]. We performed receiver operator characteristic curve analysis using time-dependent Cox proportional-hazards regression 
comparing different primary tumor size cut-points for OS at 5 years post orchiectomy. Predictive accuracy was evaluated by areas under the curve.

The optimal primary tumor size cut-point value was subsequently used to dichotomize patients into those with small vs. large primary tumors. The standardized differences approach was used to compare covariates between patients with large primary tumors managed with AS vs. AT. A standardized difference greater than $10 \%$ for a given covariate was considered a significant imbalance. Differences in baseline characteristics between patients with large primary tumors managed with AS vs. AT were controlled for with inverse probability of treatment weighting (IPTW) adjusted analyses in order to limit selection bias [24]. IPTW-adjusted Kaplan-Meier curves with IPTW-adjusted log-rank testing were used to compare OS for patients with large primary tumors treated with AT vs. AS [25]. IPTWadjusted Cox regression analysis was used to calculate the IPTW-adjusted hazard ratio (HR) of the AT effect on OS in patients with large primary tumors. Statistical analyses were performed using SAS 9.4 (SAS Institute Inc., Cary, N.C.). Two-sided statistical significance was defined as $P<0.05$.

\section{Results}

There were 2,534 (47.4\%) pT1a and 2811 (52.6\%) pT1b patients. Overall, 2,455 (47.7\%) and 2,685 (52.3\%) CS IA seminoma patients were treated with AS and AT, respectively. In the AT group, 671 (25.0\%) men were treated with adjuvant chemotherapy and 2014 (75.0\%) with adjuvant radiation.

Unweighted baseline patient characteristics stratified according to pathologic $\mathrm{T}$ stage are reported in Table 1. There were significant differences between groups with respect to several patient-level, socioeconomic and facility characteristics. pT1b patients had a larger median primary tumor size $(4.9 \mathrm{~cm}$ vs. $2.0 \mathrm{~cm}, P<0.001)$ and more often received AT (55.3\% vs. $49.1 \%, P<0.001)$ compared to pTla patients.

\subsection{Treatment trends}

Management trends for CS IA seminoma are shown in Fig. 2. Compared to AS, the use of AT decreased over the time period (annual percent change $=-2.2 ; 95 \%$ CI: -3.0 to $-1.4, P<$ 0.001). In regression multivariable logistic analysis, pT1b patients had increased odds of receiving AT (OR=1.29; 95\% CI: 1.15-1.46, $P<0.001$; Table 2). Other independent predictors of AT receipt are shown in Table 2.

\subsection{Predictors of survival}

Median follow-up for CS IA seminoma patients was 53.9 months (interquartile ranges, 28.9-83.3 months). There were 95 deaths during the study period $(4,781$ men with survival data were included in the models). Five-year OS was $98.3 \%$ and $97.5 \%$ for pT1a and pT1b patients, respectively (log-rank $P=0.005$; Supplementary Fig. 1). In unadjusted Cox regression analysis, pT1b patients had an increased risk of overall mortality (HR $=1.76$, 95\% CI: $1.18-2.65, P=0.006)$, though this association did not reach significance in multivariable analysis (HR $=1.49,95 \%$ CI: $0.97-2.28, P=0.069)$ (Table 3). Independent predictors of OS are shown in Table 3. 
Five-year OS in pT1b patients was $97.8 \%$ and $96.4 \%$ in the adjuvant and the surveillance groups, respectively (log-rank $P=0.078$; Supplementary Fig. 2). Likewise, there was no significant difference in OS between pT1b patients managed with AT compared to AS in Cox regression analyses $(P=0.19)$.

\subsection{Primary tumor size cut-point analysis}

The optimal primary tumor size cut-point for 5-year OS was $4.5 \mathrm{~cm}$ (Q statistic $=2.20, P<$ $0.001)$. Patients were further dichotomized based on this threshold $(<4.5 \mathrm{~cm}$ vs. $\geq 4.5 \mathrm{~cm})$. Supplementary Figure 3 illustrates the receiver operator characteristic curves predicting OS at 5 years following orchiectomy for different primary tumor size thresholds. areas under the curve were $0.572,0.627,0.643,0.585$ and 0.563 for the $3.0 \mathrm{~cm}, 4.0 \mathrm{~cm}, 4.5 \mathrm{~cm}, 6.0 \mathrm{~cm}$ and $7.0 \mathrm{~cm}$ cut points, respectively.

Five-year OS was $98.5 \%$ and $96.0 \%$ for patients with small and large primary tumors ( $\geq 4.5$ $\mathrm{cm}$ ), respectively (log-rank $P<0.001$; Fig. 3). In multivariable analysis, patients with large primary tumors had an increased risk of overall mortality (HR $=1.87,95 \%$ CI: $1.24-2.82, P$ $=0.003)$.

\subsection{Effectiveness of adjuvant therapy for large primary tumors}

After IPTW adjustment, all standardized differences of weighted comparisons were $<10 \%$ indicating that the distribution of the measured baseline characteristics in patients with large primary tumors was similar between the adjuvant and the surveillance groups (Supplementary Table 1).

IPTW-adjusted Kaplan-Meier curves revealed that 5-year and 10-year OS in patients with large primary tumors were $97.2 \%$ and $93.5 \%$ in the AT group vs. $93.7 \%$ and $89.0 \%$ in the AS group (IPTW-adjusted log-rank $P=0.029 ;$ Fig. 4). In IPTW-adjusted Cox regression analysis AT was associated with a significant OS benefit in patients with large primary tumors $(\mathrm{HR}=0.59,95 \% \mathrm{CI}: 0.39-0.91, P=0.017)$.

\section{Discussion}

The prognostic value of primary tumor size in pure testicular seminoma remains controversial. Using a hospital-based registry capturing over $70 \%$ of incident cancers in the US, we attempted to assess primary tumor size as a prognosticator for OS in CS IA seminoma [20]. We then determined an optimal primary tumor size cut-point and evaluated the comparative effectiveness of AS vs. AT in individuals with large T1 seminomas.

Our study revealed several noteworthy findings. First, using the AJCC $3 \mathrm{~cm}$ cut-point to subdivide CS IA seminoma patients, we did not find a significant difference in OS between pT1a and pT1b individuals. Second, we found that pT1b patients had greater odds of receiving AT compared to their pT1a counterparts. However, AT did not provide a survival benefit in pT1b patients when compared to AS. Third, we found that a primary tumor size of $\geq 4.5 \mathrm{~cm}$ was an independent predictor of inferior OS in CS IA patients. Finally, IPTWadjusted analyses revealed a significant survival benefit for AT in patients with large primary tumors. Individuals with large T1 seminomas managed with adjuvant radiation or 
chemotherapy vs. observation had a $41 \%$ decrease in overall mortality translating into a $3.5 \%$ and $4.5 \%$ absolute improvement in OS at 5 and 10 years, respectively.

There are several reports on the prognostic significance of primary tumor size on the risk of relapse in patients with CS I seminoma. Warde et al. originally used a $4 \mathrm{~cm}$ cut-point to demonstrate a non-significant difference in 5-year relapse-free rates: $88 \%$ vs. $73 \%$ for $\leq 4 \mathrm{~cm}$ vs. $>4 \mathrm{~cm}$ tumors, respectively $(P=0.12)$. A recent systematic review by a Testicular Cancer Guidelines Panel assessed 20 studies and concluded that there was a lack of evidence to justify a 4-cm cut-off although the authors did not perform a meta-analysis due to data heterogeneity [5].

The AJCC TNM staging system now subcategorizes pT1 seminoma into pT1a and pT1b using a $3 \mathrm{~cm}$ cut-point for patients with CS IA seminoma [20]. Three previous studies have reported outcomes using a $3 \mathrm{~cm}$ primary tumor size cut-point $[8,14,17]$. von der Maase et al. first reported primary tumor size as an independent prognosticator in a Danish cohort of 261 men with 4-year relapse-free survivals of 94,82 and $64 \%$ for tumors $<3, \geq 3$ to $<6$ and $\searrow 6$ $\mathrm{cm}$, respectively $(P<0.001)$ [8]. The authors concluded that men with tumors $\varangle 6 \mathrm{~cm}$ would be managed with adjuvant radiation while those with tumors $<6 \mathrm{~cm}$ would be offered surveillance. Similarly, a study on 685 patients by Chung et al. evaluating various cut-points revealed a HR for relapse of 1.87 (95\% CI: $1.15-3.06 ; P=0.01)$ in individuals with testicular seminomas $3 \mathrm{~cm}^{14}$. More recently, Nayan et al. reported a 5-year conditional risk of relapse at diagnosis of $12.2 \%$ vs. $20.3 \%$ for $\leq 3 \mathrm{~cm}$ vs. $>3 \mathrm{~cm}$ tumors ( $P$ value not reported) [17].

Per National Comprehensive Cancer Network (NCCN) Clinical Practice Guidelines, management options for men with CS IA pure seminoma include post-orchiectomy surveillance (category 1), adjuvant chemotherapy with 1-2 cycles of single-agent carboplatin or adjuvant radiation with $20 \mathrm{~Gy}$ (preferred) or $25.5 \mathrm{~Gy}$. In light of the potential long-term complications of AT compared to the low risk of recurrence, the National Comprehensive Cancer Network Panel prefers AS for CS I seminoma patients able to comply with observation strategies [26]. The potential harms of adjuvant therapies need to be disclosed to patients when making recommendations regarding management of CS IA seminoma. Several noncontemporary series have reported a significant increased risk of secondary malignancies in seminoma patients treated with radiotherapy [27,28]. Adjuvant radiation may also be associated with an increased risk of cardiovascular disease in this patient population $[28,29]$. Secondary cancers and cardiovascular events were also reported in testicular germ cell tumor patients treated with adjuvant platinum-based chemotherapy although it is unknown whether a single-agent carboplatin regimen increases such risks [29].

There is growing evidence to suggest a nonrisk-adapted approach in the management of patients with CS I testicular cancer to minimize overtreatment and avoid long-term complications associated with chemotherapy and radiation [21]. However, our findings show a near 5\% absolute OS improvement at 10 years in CS IA seminoma patients with large primary tumors managed with AT vs. AS. Although the magnitude of the survival benefit may seem marginal, it is similar to that observed in other disease settings for which neoadjuvant and/or adjuvant therapies are advocated, such as with neoadjuvant 
chemotherapy in invasive bladder cancer and adjuvant hormonal therapy in breast cancer [30,31].

This study is not without limitations. There is potential for missing data, data miscoding and unmeasured confounding factors. We performed propensity score-weighted analyses to address and limit the inherent treatment-related selection biases. The NCDB does not capture treatment information such as chemotherapy regimen or number of cycles ( $1 \mathrm{vs} .2$ cycles of single-agent Carboplatin vs. other regimens) or radiation dosing (20 vs. 25.5 Gy or more). Moreover, cancer control outcomes including cancer-specific mortality, recurrence and relapse management are not reported in the NCDB. Furthermore, the NCDB does not include information on rete testis invasion; however two recent systemic reviews concluded that its prognostic significance is limited in stage I seminoma [5,6]. With the lack of data on relapse-free survival, we investigated OS as an endpoint to assess the prognostic significance of primary tumor size. Similarly, several recently published studies on stage I testicular seminoma have used OS as a primary endpoint [32-34]. These limitations mandate caution in the interpretation of our findings especially for a malignancy with such an excellent prognosis.

Despite these limitations, our study contributes to the understanding of the prognostic value of primary tumor size in CS IA seminoma and more so the potential impact of AT on survival in individuals with large primary tumors while recognizing the trend towards a nonrisk adapted surveillance. Additionally, our study is the first attempt to validate the 8th edition of the TNM staging system for CS IA seminoma.

\section{Conclusions}

In this NCDB analysis, primary tumor size (using a $4.5 \mathrm{~cm}$ threshold) was an independent predictor of OS in CS IA testicular seminoma. The receipt of AT after orchiectomy in patients with large primary tumors $(\geq 4.5 \mathrm{~cm}$ ) was associated with a significant $O S$ benefit. These findings may warrant refinement of the AJCC staging system.

\section{Supplementary Material}

Refer to Web version on PubMed Central for supplementary material.

\section{Funding:}

This research did not receive any specific grant from funding agencies in the public, commercial, or not-for-profit sectors

\section{References}

[1]. Siegel RL, Miller KD, Jemal A. Cancer statistics, 2018. CA Cancer J Clin 2018;68:7. [PubMed: 29313949]

[2]. Znaor A, Lortet-Tieulent J, Jemal A, et al. International variations and trends in testicular cancer incidence and mortality. Eur Urol 2014;65:1095. [PubMed: 24268506]

[3]. Kollmannsberger C, Tandstad T, Bedard PL, et al. Patterns of relapse in patients with clinical stage I testicular cancer managed with active surveillance. J Clin Oncol 2015;33:51. [PubMed: 25135991] 
[4]. Warde P, Specht L, Horwich A, et al. Prognostic factors for relapse in stage I seminoma managed by surveillance: a pooled analysis. J Clin Oncol 2002;20:4448. [PubMed: 12431967]

[5]. Boormans JL, Mayor de Castro J, Marconi L, et al. Testicular tumour size and rete testis invasion as prognostic factors for the risk of relapse of clinical stage I seminoma testis patients under surveillance: a systematic review by the testicular cancer guidelines panel. Eur Urol 2017;73(3):394-405. [PubMed: 29100813]

[6]. Zengerling F, Kunath F, Jensen K, et al. Prognostic factors for tumor recurrence in patients with clinical stage I seminoma undergoing surveillance-a systematic review. Urol Oncol 2017;36(10):448-58. [PubMed: 28712790]

[7]. Warde PR, Gospodarowicz MK, Goodman PJ, et al. Results of a policy of surveillance in stage I testicular seminoma. Int J Radiat Oncol Biol Phys 1993;27:11. [PubMed: 8365931]

[8]. von der Maase H, Specht L, Jacobsen GK, et al. Surveillance following orchidectomy for stage I seminoma of the testis. Eur J Cancer 1993;29A:1931. [PubMed: 8280484]

[9]. Aparicio J, Garcia del Muro X, Maroto P, et al. Multicenter study evaluating a dual policy of postorchiectomy surveillance and selective adjuvant single-agent carboplatin for patients with clinical stage I seminoma. Ann Oncol 2003;14:867. [PubMed: 12796024]

[10]. Aparicio J, Maroto P, del Muro XG, et al. Risk-adapted treatment in clinical stage I testicular seminoma: the third Spanish germ cell cancer group study. J Clin Oncol 2011;29:4677. [PubMed: 22042940]

[11]. Tandstad T, Smaaland R, Solberg A, et al. Management of seminomatous testicular cancer: a binational prospective population-based study from the Swedish norwegian testicular cancer study group. J Clin Oncol 2011;29:719. [PubMed: 21205748]

[12]. Aparicio J, Maroto P, Garcia del Muro X, et al. Prognostic factors for relapse in stage I seminoma: a new nomogram derived from three consecutive, risk-adapted studies from the Spanish germ cell cancer group (SGCCG). Ann Oncol 2014;25:2173. [PubMed: 25210015]

[13]. Soper MS, Hastings JR, Cosmatos HA, et al. Observation versus adjuvant radiation or chemotherapy in the management of stage I seminoma: clinical outcomes and prognostic factors for relapse in a large US cohort. Am J Clin Oncol 2014;37:356. [PubMed: 23275274]

[14]. Chung P, Daugaard G, Tyldesley S, et al. Evaluation of a prognostic model for risk of relapse in stage I seminoma surveillance. Cancer Med 2015;4:155. [PubMed: 25236854]

[15]. Dieckmann KP, Dralle-Filiz I, Matthies C, et al. Testicular seminoma clinical stage 1: treatment outcome on a routine care level. J Cancer Res Clin Oncol 2016;142:1599. [PubMed: 27116691]

[16]. Tandstad T, Stahl O, Dahl O, et al. Treatment of stage I seminoma, with one course of adjuvant carboplatin or surveillance, risk-adapted recommendations implementing patient autonomy: a report from the Swedish and Norwegian Testicular Cancer Group (SWENOTECA). Ann Oncol 2016;27:1299. [PubMed: 27052649]

[17]. Nayan M, Jewett MA, Hosni A, et al. Conditional risk of relapse in surveillance for clinical stage I testicular cancer. Eur Urol 2017;71:120. [PubMed: 27527805]

[18]. Warde P, Gospodarowicz MK, Banerjee D, et al. Prognostic factors for relapse in stage I testicular seminoma treated with surveillance. J Urol 1997;157:1705. [PubMed: 9112510]

[19]. Parker C, Milosevic M, Panzarella T, et al. The prognostic significance of the tumour infiltrating lymphocyte count in stage I testicular seminoma managed by surveillance. Eur J Cancer 2002;38:2014. [PubMed: 12376206]

[20]. Paner GP, Stadler WM, Hansel DE, et al. Updates in the eighth edition of the tumor-nodemetastasis staging classification for urologic cancers. Eur Urol 2018;73:560. [PubMed: 29325693]

[21]. Pierorazio PM, Albers P, Black PC, et al. Non-risk-adapted surveillance for stage I testicular cancer: critical review and summary. Eur Urol 2018;73(6):899-907. [PubMed: 29338978]

[22]. Winchester DP, Stewart AK, Bura C, et al. The National cancer data base: a clinical surveillance and quality improvement tool. J Surg Oncol 2004;85:1. [PubMed: 14696080]

[23]. Contal C, O'Quingley J. An application of changepoint methods in studying the effect of age on survival in breast cancer. Comput Stat Data An 1999;30:253. 
[24]. Austin PC. The use of propensity score methods with survival or time-to-event outcomes: reporting measures of effect similar to those used in randomized experiments. Stat Med 2014;33:1242. [PubMed: 24122911]

[25]. Cole SR, Hernan MA. Adjusted survival curves with inverse probability weights. Comput Methods Programs Biomed 2004;75:45. [PubMed: 15158046]

[26]. Motzer RJ, Jonasch E, Agarwal N, et al. Testicular cancer, version 2.2015. J Natl Compr Canc Netw 2015;13:772. [PubMed: 26085393]

[27]. Travis LB, Fossa SD, Schonfeld SJ, et al. Second cancers among 40,576 testicular cancer patients: focus on long-term survivors. J Natl Cancer Ins 2005;97:1354.

[28]. Beard CJ, Travis LB, Chen MH, et al. Outcomes in stage I testicular seminoma: a populationbased study of 9193 patients. Cancer 2013;119:2771. [PubMed: 23633409]

[29]. Huddart RA, Norman A, Shahidi M, et al. Cardiovascular disease as a long-term complication of treatment for testicular cancer. J Clin Oncol 2003;21:1513. [PubMed: 12697875]

[30]. Advanced Bladder Cancer Meta-analysis, C. Neoadjuvant chemotherapy in invasive bladder cancer: update of a systematic review and meta-analysis of individual patient data advanced bladder cancer (ABC) meta-analysis collaboration. Eur Urol 2005;48:202. [PubMed: 15939524]

[31]. Glassman D, Hignett S, Rehman S, et al. Adjuvant endocrine therapy for hormone-positive breast cancer, focusing on ovarian suppression and extended treatment: an update. Anticancer Res 2017;37:5329. [PubMed: 28982841]

[32]. Gray PJ, Lin CC, Sineshaw H, et al. Management trends in stage I testicular seminoma: Impact of race, insurance status, and treatment facility. Cancer 2015;121:681. [PubMed: 25345675]

[33]. Kamran SC, Seisen T, Markt SC, et al. Contemporary treatment patterns and outcomes for clinical stage Is testicular cancer. Eur Urol 2018;73:262. [PubMed: 28687143]

[34]. Glaser SM, Vargo JA, Balasubramani GK, et al. Stage II testicular seminoma: patterns of care and survival by treatment strategy. Clin Oncol (R Coll Radiol) 2016;28:513. [PubMed: 27146264]

Urol Oncol. Author manuscript; available in PMC 2021 January 01. 


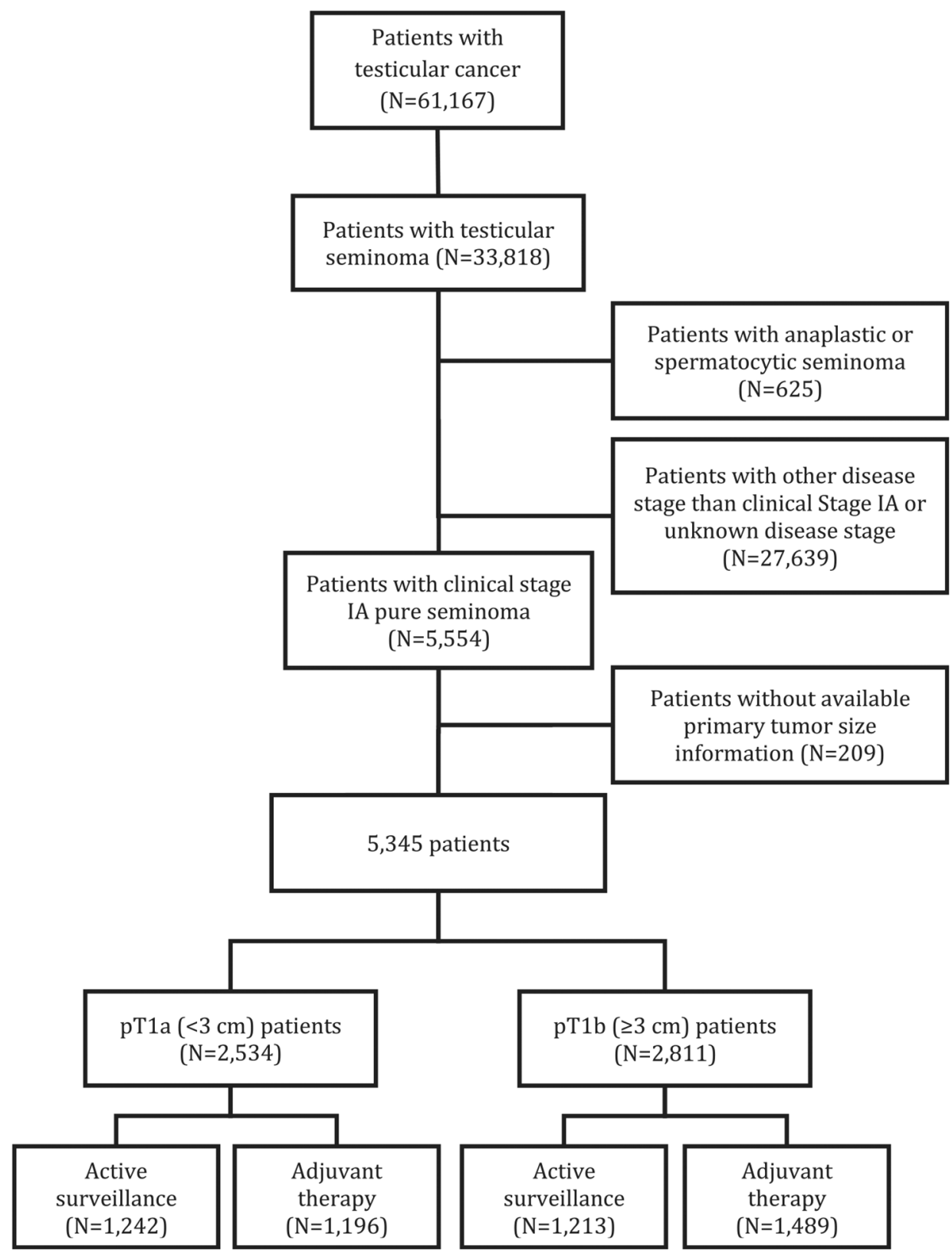

Fig. 1.

Flowchart describing the selection of clinical stage IA testicular seminoma patients in the National Cancer Data Base, 2004 to 2014. 


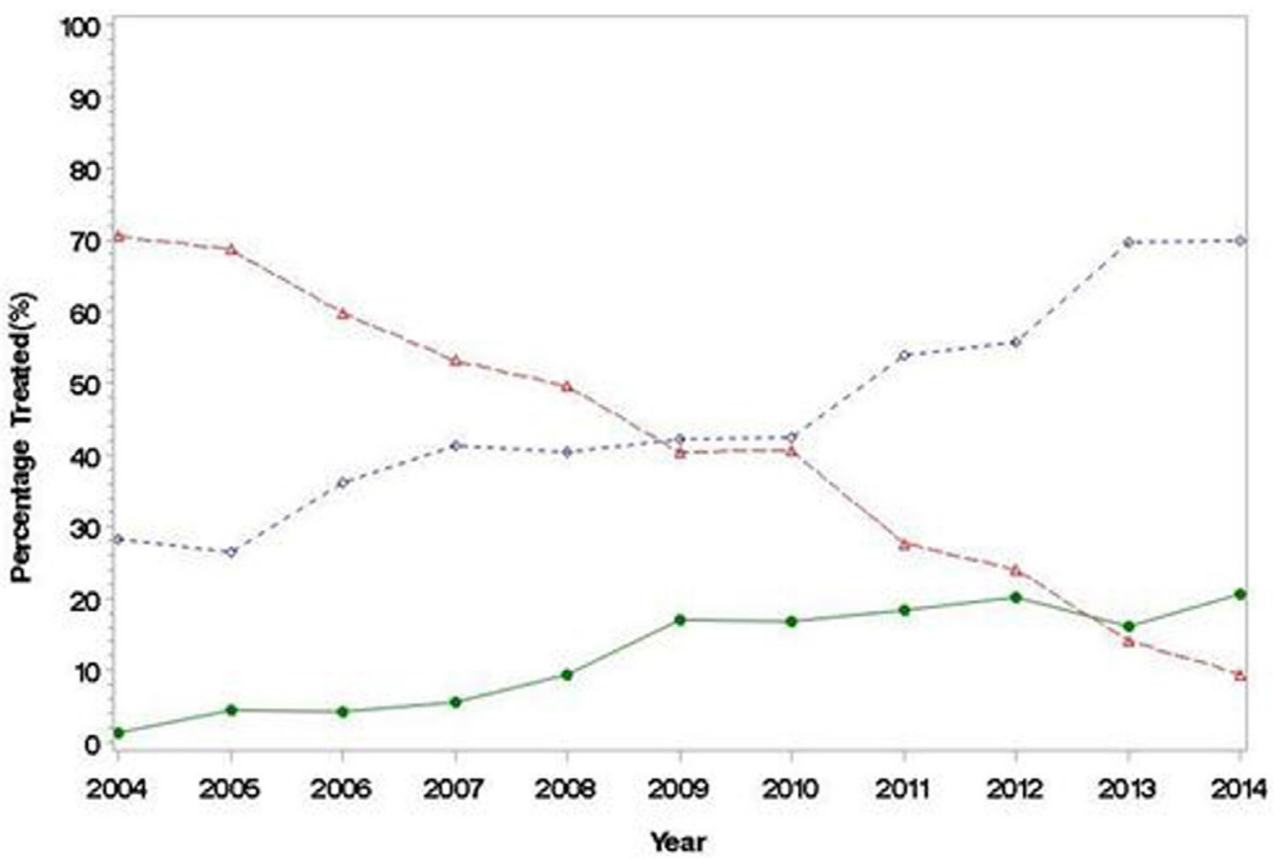

\begin{tabular}{|l|c|c|c|c|c|c|c|c|c|c|c|}
\hline & 2004 & 2005 & 2006 & 2007 & 2008 & 2009 & 2010 & 2011 & 2012 & 2013 & 2014 \\
\hline Chemotherapy & 5 & 18 & 16 & 21 & 37 & 78 & 87 & 91 & 115 & 89 & 114 \\
\hline Observation & 119 & 110 & 141 & 156 & 160 & 193 & 220 & 269 & 318 & 385 & 384 \\
\hline Radiation & 297 & 285 & 234 & 201 & 196 & 185 & 211 & 138 & 137 & 78 & 52 \\
\hline
\end{tabular}

Fig. 2.

Temporal treatment trends for clinical stage IA testicular seminoma patients in the National Cancer Database, 2004 to 2014. 


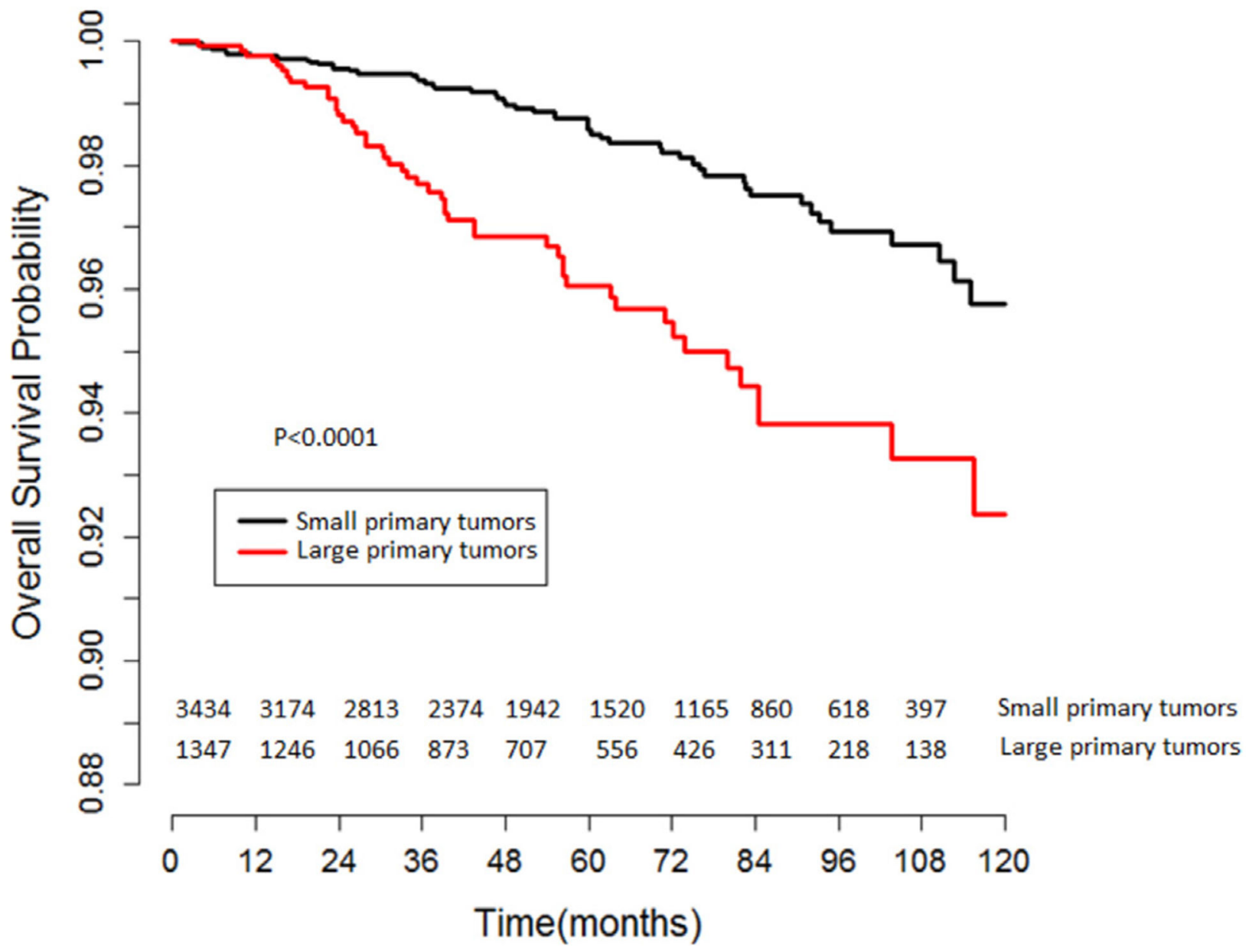

Fig. 3.

Unadjusted Kaplan-Meier analysis of overall survival in stage IA testicular seminoma patients stratified by primary tumor size (small vs. large primary tumors using a $4.5 \mathrm{~cm}$ threshold). 


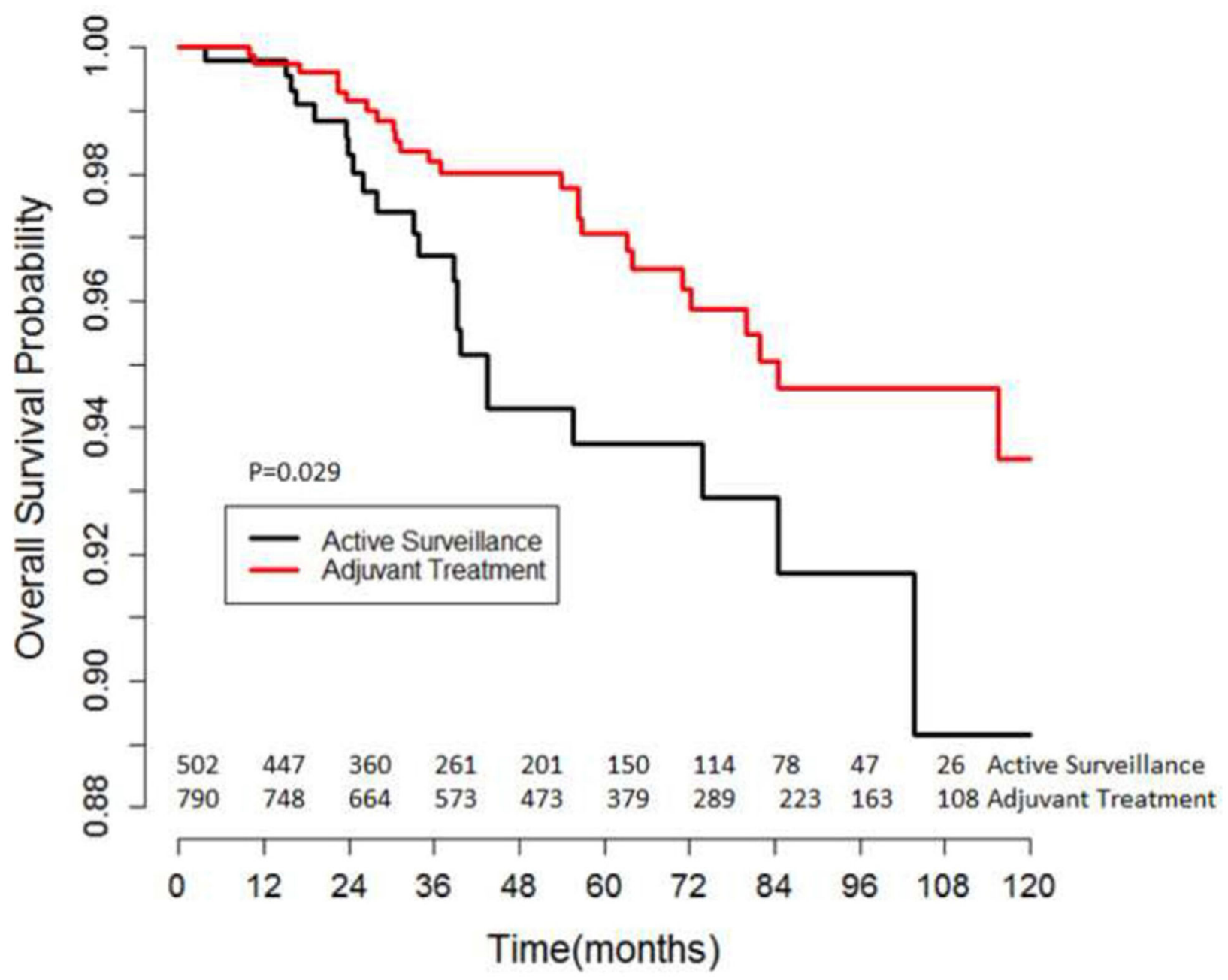

Fig. 4.

Inverse-probability of treatment weighting-adjusted Kaplan-Meier analysis of overall survival in stage IA testicular seminoma patients with large primary tumors ( $\geq 4.5 \mathrm{~cm}$ ) managed with active surveillance vs. adjuvant therapy. 


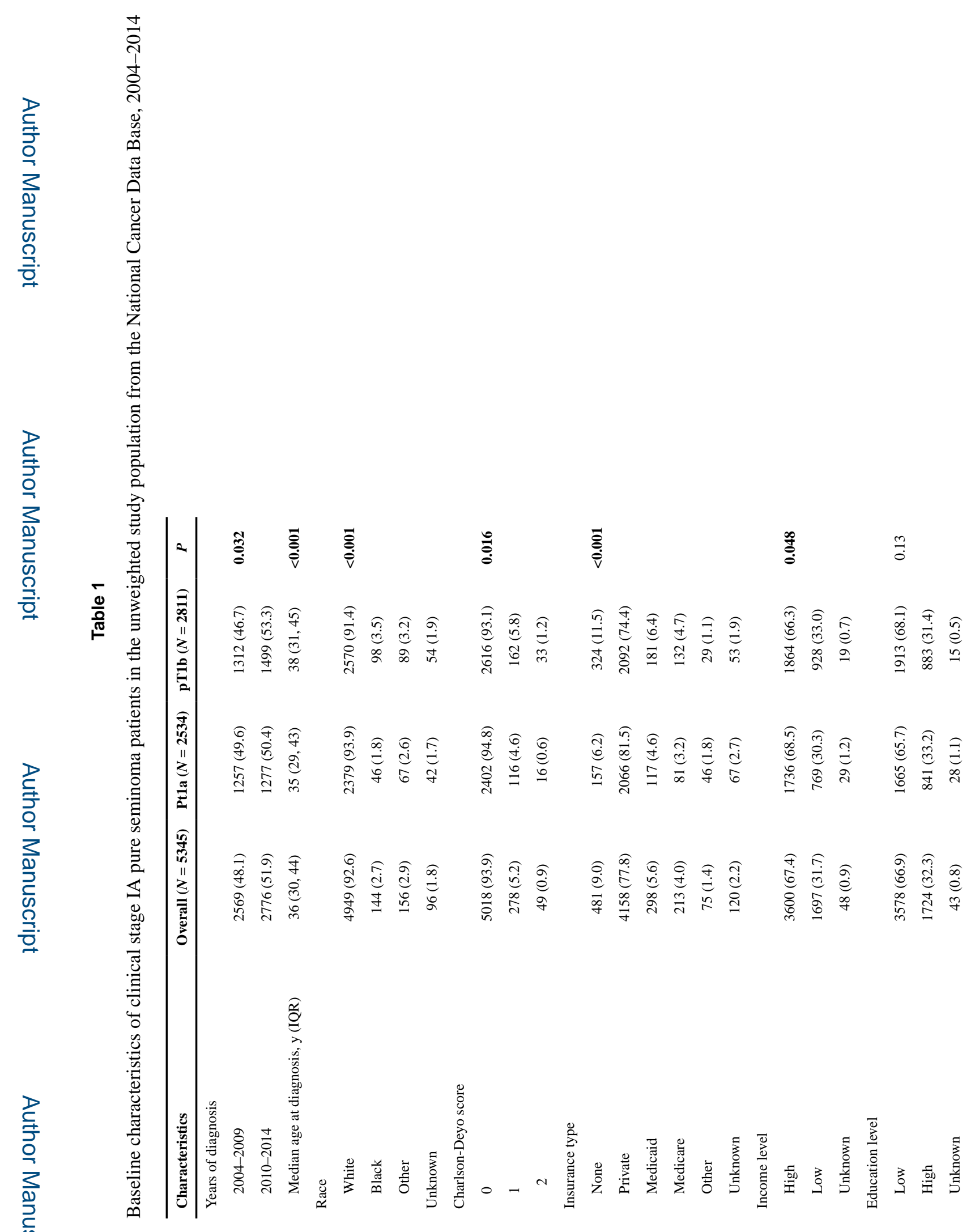

Urol Oncol. Author manuscript; available in PMC 2021 January 01. 


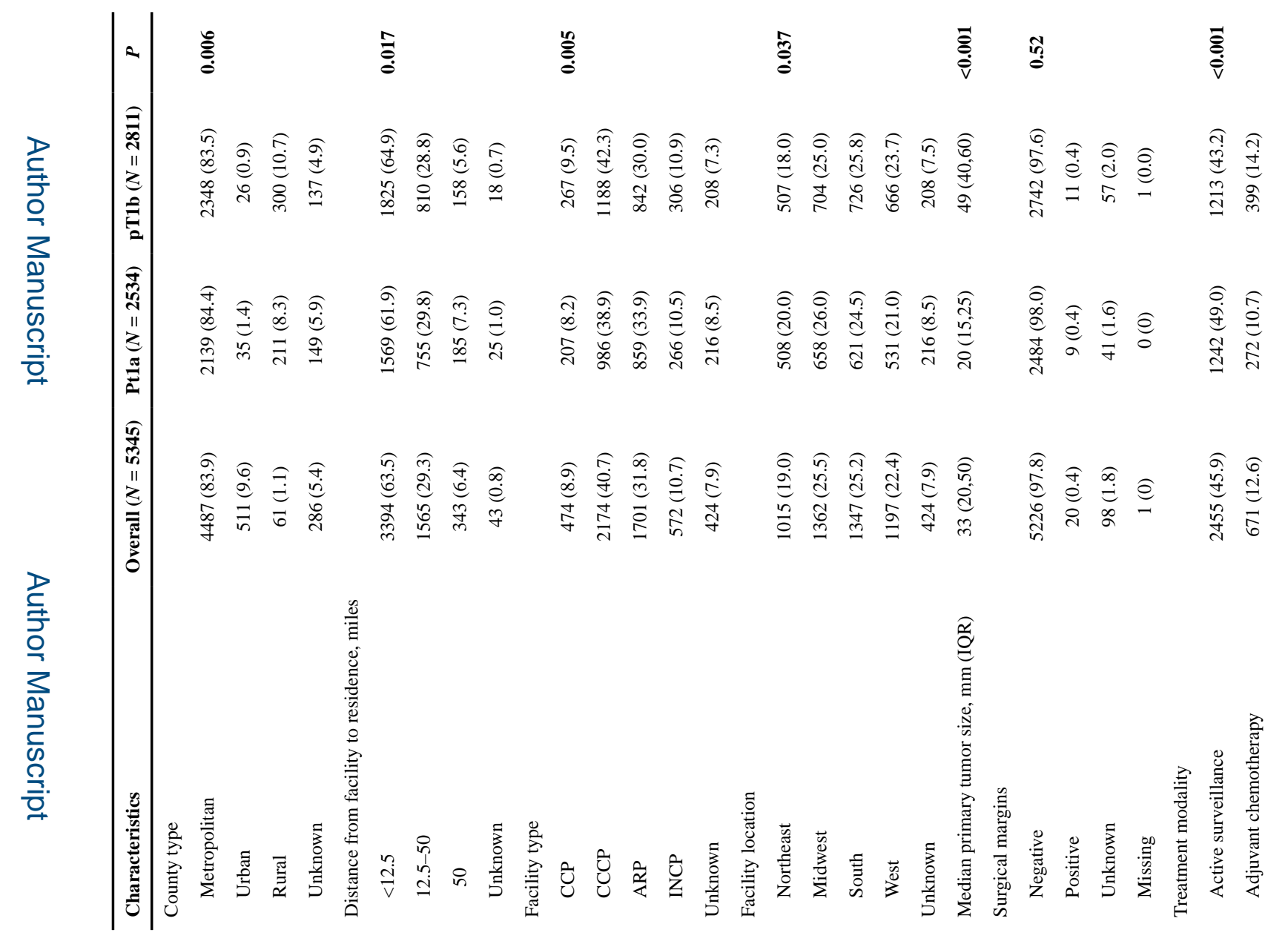

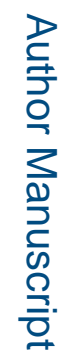

로을

Urol Oncol. Author manuscript; available in PMC 2021 January 01. 


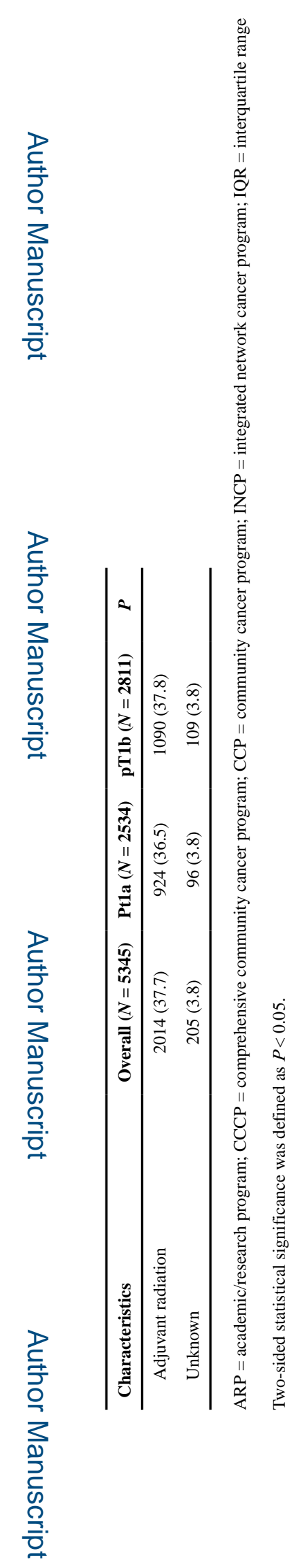

Urol Oncol. Author manuscript; available in PMC 2021 January 01. 


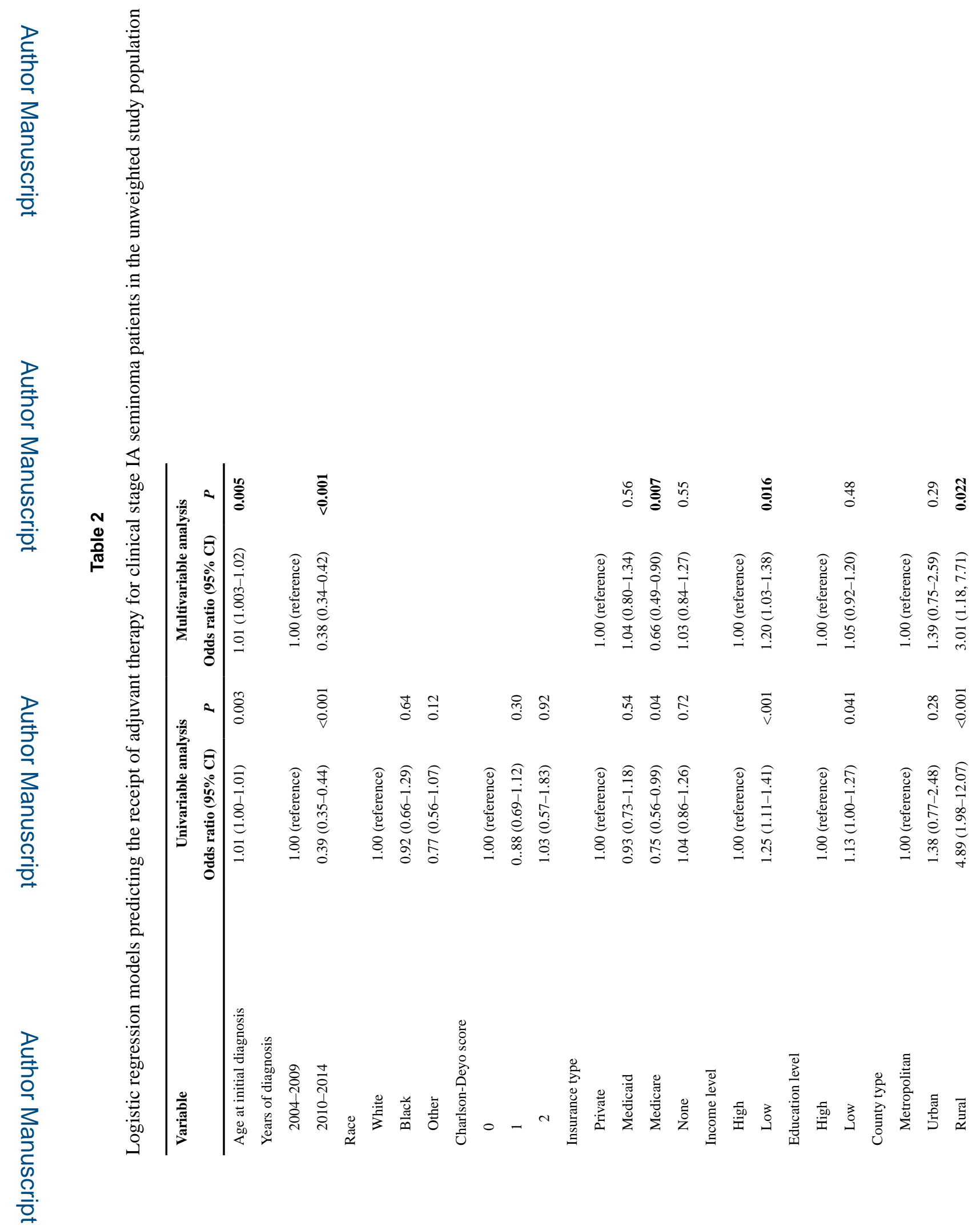

Urol Oncol. Author manuscript; available in PMC 2021 January 01. 


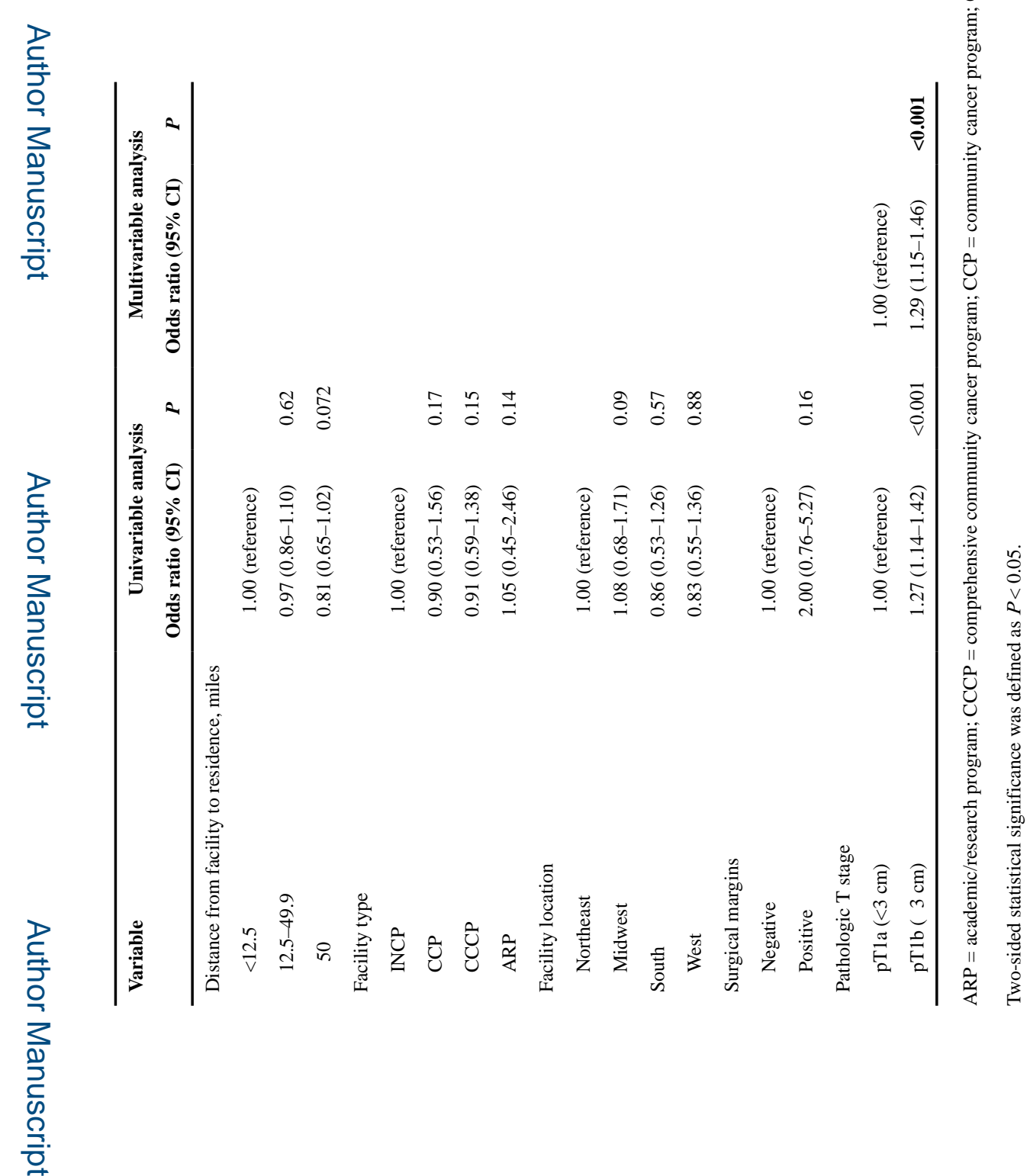




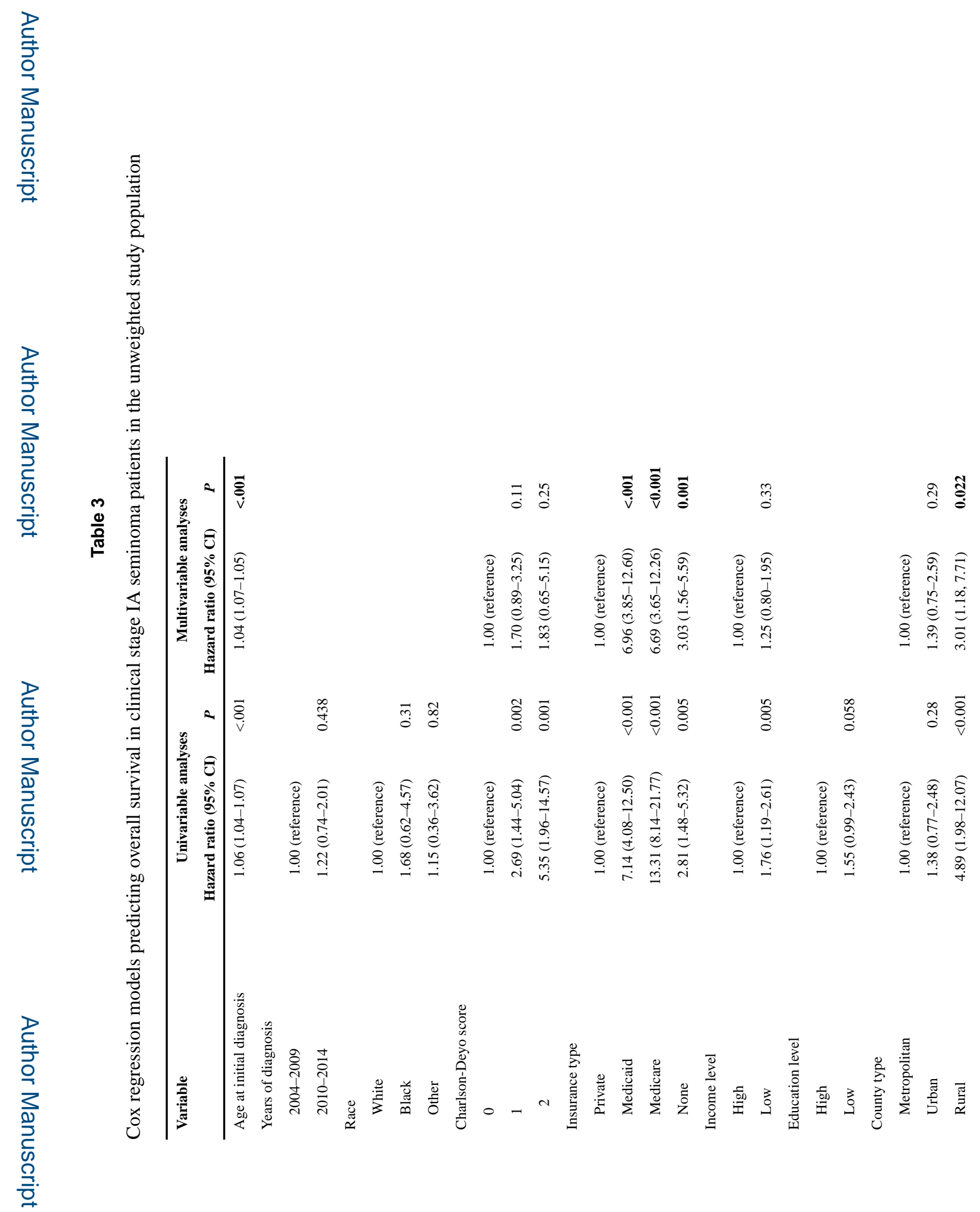

Urol Oncol. Author manuscript; available in PMC 2021 January 01. 


\section{를 \\ 일}

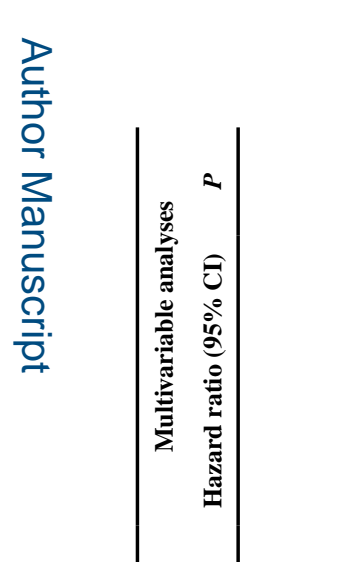

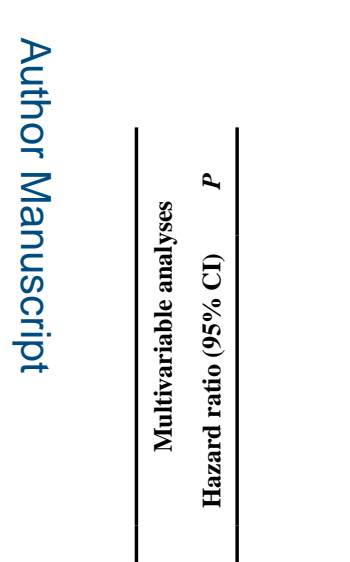

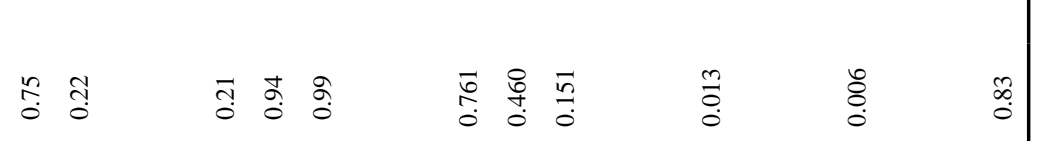

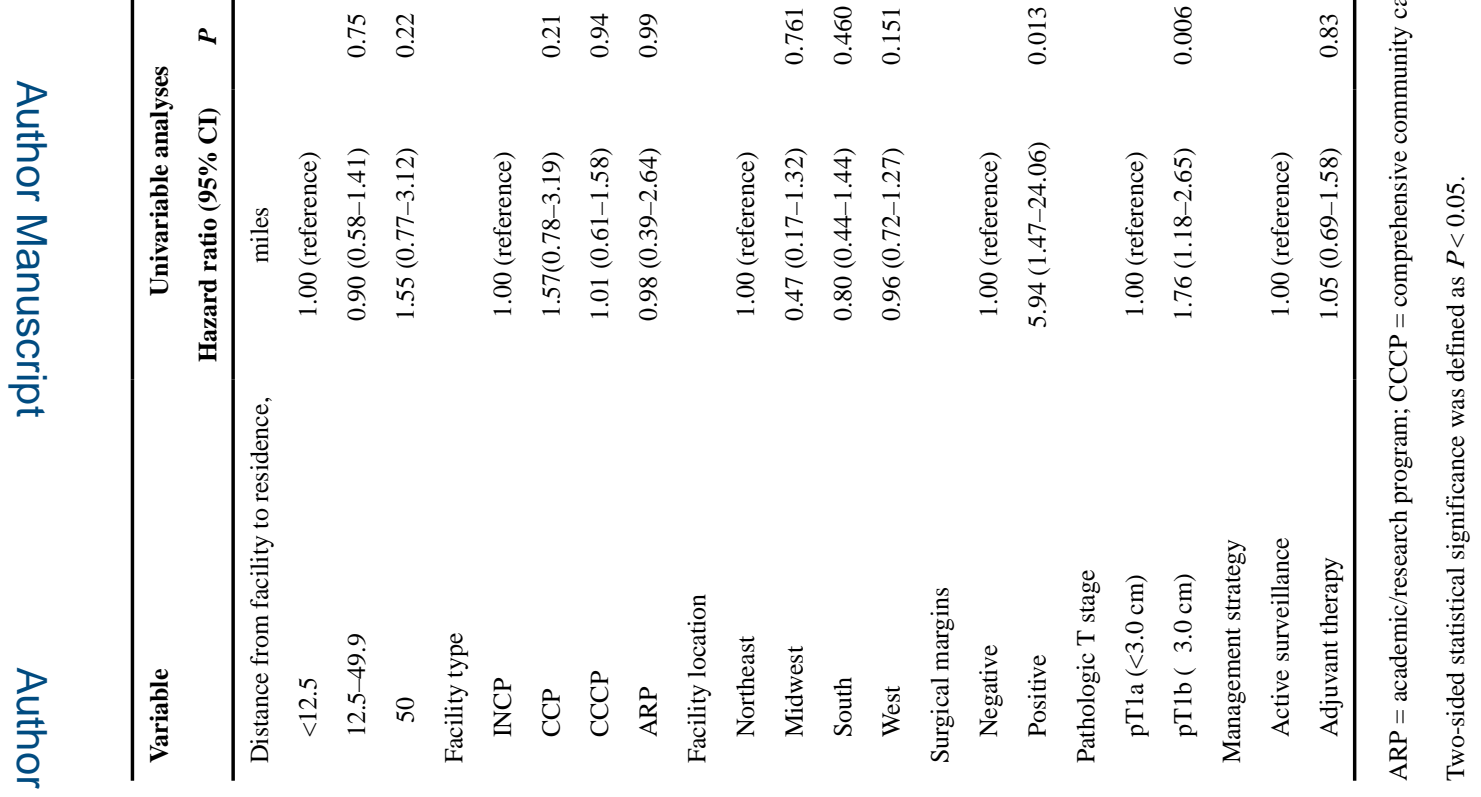

3
$\frac{1}{2}$
$\frac{1}{0}$
$\frac{9}{\bar{D}}$
$\frac{9}{0}$

Urol Oncol. Author manuscript; available in PMC 2021 January 01. 